\title{
Choroid plexus differences in rats with spontaneous and induced hydrocephalus
}

\author{
Agustín Castañeyra-Perdomo*1,2, Ibrahim González-Marrero², \\ Juan M González-Toledo², Leandro Castañeyra-Ruiz' ${ }^{1}$, Héctor de Paz- \\ Carmona ${ }^{1}$, Agustín Castañeyra-Ruiz ${ }^{1}$, Maria Castañeyra ${ }^{1}$, Isabel Hernández- \\ Garde $^{2}$ and Emilia M Carmona-Calero ${ }^{1,2}$
}

\author{
Address: ${ }^{1}$ Departamento de Biotecnología, Instituto de Investigación y Ciencias de Puerto del Rosario, Fuerteventura, Spain and ${ }^{2}$ Departamento \\ de Anatomía, Facultad de Medicina, Universidad de La Laguna, Tenerife, Spain \\ Email: Agustín Castañeyra-Perdomo* - acastane@ull.es \\ * Corresponding author
}

from 53rd Annual Meeting of the Society for Research into Hydrocephalus and Spina Bifida Belfast, UK. 24-27 June 2009

Published: 27 November 2009

Cerebrospinal Fluid Research 2009, 6(Suppl 2):S42 doi:10.1186/I743-8454-6-S2-S42

This abstract is available from: http://www.cerebrospinalfluidresearch.com/content/6/S2/S42

(C) 2009 Castañeyra-Perdomo et al; licensee BioMed Central Ltd.

\section{Background}

In hydrocephalus alterations of the brain structures have been described, among them: cortical displacement, cerebrospinal fluid composition alterations and circumventricular structure variations are described and many of these variations could be cause or consequences of the hydrocephalus. The protein p73 is a member of a family of transcription factors and equilibrium between $\mathrm{p} 73$ isoforms is necessary for the normal development of the brain and the maturation of the neuroepithelium. On the other hand, the p73 deficiency produces many alterations in the brain that include hydrocephalus. The purpose of this work was to analyze the alteration of the choroid plexus (CP) in different types of hydrocephalus.

\section{Materials and methods}

Ten control male rats and ten male rats with spontaneous and induced hydrocephalus were used. The control group was composed of rats sacrificed at 26 and 50 weeks of age and the hydrocephalic rats ( 6 kaolin-induced hydrocephalus, 4 spontaneous hydrocephalus) rats were sacrificed at the same ages as control. The rats were fixed by vascular perfusion with Bouin's fluid, dehydrated and embedded in paraffin under standard conditions. Brains were cut into four serial coronal sections. One of the serial coronal sections was stained by the Klüver-Barrera method. The other sections were immunohistochemically processed using anti-p73 and anti-transthyretin (TTR) as primary antibodies.

\section{Results}

We found, in the induced hydrocephalus, structural alterations and a decrease in the p73 immunoreactive material when compared to control group. In spontaneous hydrocephalus we did not find great structural variations of the $\mathrm{CP}$, however changes in the intensity of anti-p73 reaction were found with respect to the control. The TTR expression varied with the hydrocephalus, in control rats it was clearly observed in the CP and was increased in spontaneous hydrocephalus.

\section{Conclusion}

The obstructive hydrocephalus causes CP morphology alterations and a decrease in the p73 and expression, contrarily the $\mathrm{CP}$, significant structural variations in the spontaneous hydrocephalus were not found. Furthermore, some changes in the intensity of anti-p73 and anti-TTR were found and these variations could precede the onset of the hydrocephalus. 